\title{
Fixed point theorems for a new nonlinear mapping similar to a nonspreading mapping
}

Tomonari Suzuki*

Dedicated to Professor Wataru Takahashi on the occasion of his 70th birthday.

"Correspondence:

suzuki-t@mns.kyutech.ac.jp Department of Basic Sciences, Kyushu Institute of Technology, Tobata, Kitakyushu, 804-8550, Japan

\begin{abstract}
We introduce a new nonlinear mapping similar to the nonspreading mapping which is defined by Kohsaka and Takahashi (Arch. Math. 91:166-177, 2008). We prove fixed point theorems for such a mapping without the convexity of the domain. We also prove convergence theorems.

MSC: Primary 47H10; secondary 47H09; 47J25
\end{abstract}

Keywords: nonspreading mapping; Chatterjea mapping; fixed point; convergence theorem

\section{Introduction}

In 2008, Kohsaka and Takahashi [1] introduced the concept of nonspreading mapping. Let $T$ be a mapping on a subset $C$ of a smooth Banach space $E$. $T$ is said to be nonspreading if

$$
\phi(T x, T y)+\phi(T y, T x) \leq \phi(T x, y)+\phi(T y, x)
$$

for all $x, y \in C$, where $\phi(x, y)=\|x\|^{2}-2\langle x, J y\rangle+\|y\|^{2}$. We denote by $F(T)$ the set of fixed points of $T$. They proved $F(T)$ is nonempty provided $C$ is bounded, closed, and convex; and $E$ is reflexive and strictly convex. In the case where $E$ is Hilbertian, (1) is equivalent to

$$
2\|T x-T y\|^{2} \leq\|T x-y\|^{2}+\|x-T y\|^{2} .
$$

We note that the concept of nonspreading mapping is very important because of useful applications.

On the other hand, Chatterjea [2] considered the following condition:

$$
\|T x-T y\| \leq \alpha\|T x-y\|+\alpha\|x-T y\|,
$$

where $\alpha$ is constant belonging to $(0,1 / 2)$.

Motivated by the above, we introduce a new concept, named Chatterjea mapping. The condition (5) of this concept is weaker than (2). It is meaningful to study Chatterjea mapping because the concept of nonspreading mapping is very important. In this paper, we prove fixed point theorems for Chatterjea mappings without the convexity of the domain. We also prove convergence theorems to a fixed point.

o2014 Suzuki; licensee Springer. This is an Open Access article distributed under the terms of the Creative Commons Attribution License (http://creativecommons.org/licenses/by/2.0), which permits unrestricted use, distribution, and reproduction in any medium, provided the original work is properly cited. 


\section{Preliminaries}

Throughout this paper we denote by $\mathbb{N}$ the set of all positive integers and by $\mathbb{R}$ the set of all real numbers. For $n \in \mathbb{N} \cup\{0\}$, we define $n$ ! by $0 !=1$ and $(n+1) !=n !(n+1)$, that is, $n !$ is the factorial of $n$. Stirling's formula $\lim _{n} \sqrt{2 \pi n}(n / e)^{n} /(n !)=1$ is well known. For $n, k \in \mathbb{N} \cup\{0\}$ with $k \leq n$, we define $C(n, k)=n ! /(k !(n-k) !)$, that is, $C(n, k)$ is the binomial coefficient of $(n ; k)$.

A Banach space $E$ is said to be smooth if the $\operatorname{limit}_{\lim } \lim _{t \rightarrow 0}(\|x+t y\|-\|x\|) / t$ exists for each $x, y \in E$ with $\|x\|=\|y\|=1$. The normalized duality mapping $J$ from $E$ into $E^{*}$ is defined by $\langle x, J x\rangle=\|x\|^{2}=\|J x\|^{2}$ for all $x \in E$.

Let $E$ be a Banach space. $E$ is said to be strictly convex if $\|x+y\|<2$ for all $x, y \in E$ with $\|x\|=\|y\|=1$ and $x \neq y$. We recall that $E$ is said to be uniformly convex in every direction (UCED, for short) if for $\varepsilon \in(0,2]$ and $z \in E$ with $\|z\|=1$, there exists $\delta>0$ such that

$$
\|x+y\| \leq 2(1-\delta)
$$

for all $x, y \in E$ with $\|x\| \leq 1,\|y\| \leq 1$ and $x-y \in\{t z: t \in[-2,-\varepsilon] \cup[+\varepsilon,+2]\}$. It is obvious that UCED implies strictly convexity. We know that every separable Banach space can be equivalently renormed so that it is UCED. See $[3,4]$ and others. We know UCED is characterized as follows.

Lemma 1 ([5]) For a Banach space E, the following are equivalent:

(i) $E$ is UCED.

(ii) If $\left\{u_{n}\right\}$ is a bounded sequence in $E$, then a function $g$ on $E$ defined by

$$
g(x)=\limsup _{n \rightarrow \infty}\left\|u_{n}-x\right\|
$$

is strictly quasiconvex, that is,

$$
g(\lambda x+(1-\lambda) y)<\max \{g(x), g(y)\}
$$

for all $\lambda \in(0,1)$ and $x, y \in E$ with $x \neq y$.

Let $C$ be a subset of a Banach space $E$. $C$ is said to be boundedly weakly compact if its intersection with any closed ball is weakly compact. It is obvious that if $E$ is reflexive, then every closed convex subset is boundedly weakly compact. $C$ is said to have the Opial property [6] if for each weakly convergent sequence $\left\{x_{n}\right\}$ in $C$ with weak limit $z \in C$,

$$
\liminf _{n \rightarrow \infty}\left\|x_{n}-z\right\|<\liminf _{n \rightarrow \infty}\left\|x_{n}-y\right\|
$$

holds for $y \in C$ with $y \neq z$. We remark that we may replace 'lim inf' by 'lim sup'. All nonempty compact subsets have the Opial property. Also, all Hilbert spaces, $\ell^{p}(1 \leq p<\infty)$ and finite dimensional Banach spaces have the Opial property. A Banach space with a duality mapping which is weakly sequentially continuous also has the Opial property [7]. We know that every separable Banach space can be equivalently renormed so that it has the Opial property [8].

Lemma 2 Let $C$ be a boundedly weakly compact subset of a Banach space $E$ and let $f$ be a function from $C$ into $\mathbb{R}$ which is lower semicontinuous in the weak topology. Assume that 
either $C$ is bounded or $f$ satisfies

$$
\inf \{f(x): x \in C\}<\lim _{r \rightarrow \infty} \inf \{f(x): x \in C,\|x\| \geq r\} .
$$

Then $\min f(C)$ exists.

Proof Let $\left\{x_{n}\right\}$ be a sequence in $C$ such that $\lim _{n} f\left(x_{n}\right)=\inf f(C)$. From the assumption, $\left\{x_{n}\right\}$ is bounded. Since $C$ is boundedly weakly compact, there exist a subsequence $\left\{x_{n_{j}}\right\}$ of $\left\{x_{n}\right\}$ and $z \in C$ such that $\left\{x_{n_{j}}\right\}$ converges weakly to $z$. Since $f$ is lower semicontinuous in the weak topology, we have

$$
\inf f(C) \leq f(z) \leq \liminf _{j \rightarrow \infty} f\left(x_{n_{j}}\right)=\inf f(C)
$$

and hence $f(z)=\inf f(C)$.

Let $C$ be a subset of a Banach space $E$ and let $f$ be a function from $C$ into $\mathbb{R}$. $f$ is said to be nonincreasing with respect to a mapping $T$ on $C$ if $f(T x) \leq f(x)$ for all $x \in C$. Also, from now on, in the case where $C$ is bounded, we consider every function $f$ to satisfy (4).

The proof of the following lemma is obvious.

Lemma 3 Let $\eta$ be a continuous, strictly increasing function from $[0, \infty)$ into itself. Then the following hold:

(i) $s \leq t$ if and only if $\eta(s) \leq \eta(t)$.

(ii) If $\lim \sup _{n} t_{n} \in \mathbb{R}$, then $\eta\left(\lim \sup _{n} t_{n}\right)=\lim \sup _{n} \eta\left(t_{n}\right)$.

\section{Chatterjea mapping}

In this section, we introduce the concept of Chatterjea mapping.

Let $T$ be a mapping on a subset $C$ of a Banach space $E$ and let $\eta$ be a continuous, strictly increasing function from $[0, \infty)$ into itself with $\eta(0)=0$. Then $T$ is call a Chatterjea mapping with $\eta$ if

$$
2 \eta(\|T x-T y\|) \leq \eta(\|T x-y\|)+\eta(\|x-T y\|)
$$

for all $x, y \in C$.

From the definition, we can obtain the following propositions.

Proposition 4 Let $T$ be a nonspreading mapping on a subset $C$ of a Hilbert space E. Then $T$ is Chatterjea with $t \mapsto t^{2}$.

Proof Obvious.

Proposition 5 Let $T$ be a mapping on a subset $C$ of a Banach space $E$ and let $\eta$ be a continuous, strictly increasing function from $[0, \infty)$ into itself with $\eta(0)=0$. Assume that there exists $\alpha \in[0,1]$ such that

$$
\eta(\|T x-T y\|) \leq \alpha \eta(\|T x-y\|)+(1-\alpha) \eta(\|x-T y\|)
$$

for all $x, y \in C$. Then $T$ is Chatterjea with $\eta$. 
Proof For $x, y \in C$, we have

$$
\eta(\|T x-T y\|) \leq \alpha \eta(\|T x-y\|)+(1-\alpha) \eta(\|x-T y\|)
$$

and

$$
\eta(\|T y-T x\|) \leq \alpha \eta(\|T y-x\|)+(1-\alpha) \eta(\|y-T x\|) .
$$

Adding the both inequalities, we obtain the desired result.

Proposition 6 Let $p$ and $q$ be positive real numbers with $p<q$. Let $T$ be a mapping on a subset $C$ of a Banach space E. Assume $T$ is Chatterjea with $t \mapsto t^{p}$. Then $T$ is also Chatterjea with $t \mapsto t^{q}$.

Proof Let $\alpha$ and $\beta$ be nonnegative real numbers and define functions $f$ and $g$ from $(0, \infty)$ into $\mathbb{R}$ by

$$
f(r)=r \ln r \quad \text { and } \quad g(r)=\left(\alpha^{r}+\beta^{r}\right)^{1 / r} 2^{-1 / r} .
$$

Then we note $f$ is convex because $f^{\prime \prime}(r)=1 / r>0$. We next show that $g$ is nondecreasing. In the case where $\alpha=0$ or $\beta=0$, the nondecreasingness of $g$ is obvious. In the case where $\alpha>0$ and $\beta>0$, we have

$$
\begin{aligned}
\frac{d}{d r} g(r) & =g(r) \frac{d}{d r}(\ln (g(r))) \\
& =\frac{2 g(r)}{r^{2}\left(\alpha^{r}+\beta^{r}\right)}\left(\frac{f\left(\alpha^{r}\right)+f\left(\beta^{r}\right)}{2}-f\left(\frac{\alpha^{r}+\beta^{r}}{2}\right)\right) \geq 0
\end{aligned}
$$

and hence $g$ is nondecreasing. Let $x, y \in C$ be fixed. Then we have

$$
\begin{aligned}
\|T x-T y\| & \leq\left(\|T x-y\|^{p}+\|x-T y\|^{p}\right)^{1 / p} 2^{-1 / p} \\
& \leq\left(\|T x-y\|^{q}+\|x-T y\|^{q}\right)^{1 / q} 2^{-1 / q}
\end{aligned}
$$

which implies that $T$ is Chatterjea with $t \mapsto t^{q}$.

Example 7 Let $p$ and $q$ be positive real numbers with $p<q$. Let $E$ be a Banach space and let $w \in E \backslash\{0\}$ be fixed. Define a mapping $T$ on $E$ by

$$
T x= \begin{cases}0 & \text { if } x \neq w \\ 2^{-1 / q} w & \text { if } x=w .\end{cases}
$$

Then $T$ is Chatterjea with $t \mapsto t^{q}$, however, $T$ is not Chatterjea with $t \mapsto t^{p}$.

Proof Let $x \in E \backslash\{w\}$ be fixed. Then since $T x=0$, we have

$$
2\|T w-T x\|^{q}=\|w\|^{q} \leq\|T w-x\|^{q}+\|w\|^{q}=\|T w-x\|^{q}+\|w-T x\|^{q},
$$


which implies that $T$ is Chatterjea with $t \mapsto t^{q}$. On the other hand, since $T^{2} w=0$, we have

$$
2\left\|T w-T^{2} w\right\|^{p}=2^{1-p / q}\|w\|^{p}>\|w\|^{p}=\|T w-T w\|^{p}+\left\|w-T^{2} w\right\|^{p},
$$

which implies that $T$ is not Chatterjea with $t \mapsto t^{p}$.

Remark Example 7 also informs that Chatterjea mappings are not necessarily continuous.

\section{Basic properties}

In this section, we prove basic properties of Chatterjea mapping.

A mapping $T$ on a subset $C$ of a Banach space $E$ is said to be quasinonexpansive [9] if

$$
\|T x-z\| \leq\|x-z\|
$$

for all $x \in C$ and $z \in F(T)$.

Proposition 8 Assume that a mapping $T$ on $C$ is Chatterjea and has a fixed point. Then $T$ is a quasinonexpansive mapping.

Proof Let $\eta$ satisfy (5). For $x \in C$ and $z \in F(T)$, we have

$$
2 \eta(\|T x-z\|) \leq \eta(\|T x-z\|)+\eta(\|x-z\|) .
$$

Using this and the strict increasingness of $\eta$, we obtain (6).

From Proposition 8, we obtain the following.

Lemma 9 Assume that a mapping $T$ on $C$ is Chatterjea and has a fixed point. Then $\left\{T^{n} u\right\}$ is bounded for all $u \in C$.

Proposition 10 Let $T$ be a Chatterjea mapping on a closed subset $C$ of a Banach space $E$. Then $F(T)$ is closed. Moreover, if $E$ is strictly convex and $C$ is convex, then $F(T)$ is also convex.

The following lemma plays a very important role in this paper.

Lemma 11 Put $I_{0}=\{(m, n): m, n \in \mathbb{N} \cup\{0\}, m \leq n\}$ and $I=\{(m, n): m, n \in \mathbb{N}, m<n\}$. Let $A$ be a function from $I_{0}$ into $[0, \infty)$ satisfying the following:

- $A(0, n) \leq 1$ for $n \in \mathbb{N} \cup\{0\}$;

- $A(n, n)=0$ for $n \in \mathbb{N}$

- $A(m, n) \leq(1 / 2) A(m-1, n)+(1 / 2) A(m, n-1)$ for $(m, n) \in I$.

Then the following hold:

(i) $A(m, n) \leq 1$ for $(m, n) \in I_{0}$;

(ii) $A(j+2 n, j+2 n+1) \leq \frac{1}{2^{2 n}} \sum_{k=0}^{n} C(2 n, k) \frac{2 n-2 k+1}{2 n-k+1} A(j+k, j+2 n+1-k)$ for $j, n \in \mathbb{N} \cup\{0\}$;

(iii) $A(j+2 n+1, j+2 n+2) \leq \frac{1}{2^{2 n+1}} \sum_{k=0}^{n} C(2 n+1, k) \frac{2 n-2 k+2}{2 n-k+2} A(j+k, j+2 n+2-k)$ for $j, n \in \mathbb{N} \cup\{0\} ;$

(iv) $\lim _{n \rightarrow \infty} A(n, n+1)=0$. 
Proof We first note $A(m, n) \leq 1$ for $(m, n) \in I_{0} \backslash I$. Define a total order $\leq$ on $I_{0}$ by $\left(m_{1}, n_{1}\right) \leq$ $\left(m_{2}, n_{2}\right)$ iff either $n_{1}<n_{2}$ or $n_{1}=n_{2}$ and $m_{1} \leq m_{2}$. Fix $(m, n) \in I$ and assume $A\left(m^{\prime}, n^{\prime}\right) \leq 1$ for $\left(m^{\prime}, n^{\prime}\right) \in I_{0}$ with $\left(m^{\prime}, n^{\prime}\right)<(m, n)$. Then we have

$$
A(m, n) \leq(1 / 2) A(m-1, n)+(1 / 2) A(m, n-1) \leq 1 / 2+1 / 2=1 .
$$

By induction, we obtain (i). In order to show (ii) and (iii), we also use induction with respect to $n$. When $n=0$, (ii) becomes $A(j, j+1) \leq A(j, j+1)$, which clearly holds. We assume (ii) holds for some $n \in \mathbb{N} \cup\{0\}$. Then we have

$$
\begin{aligned}
A(j+ & 2 n+1, j+2 n+2) \\
= & A(j+1+2 n, j+1+2 n+1) \\
\leq & \frac{1}{2^{2 n}} \sum_{k=0}^{n} C(2 n, k) \frac{2 n-2 k+1}{2 n-k+1} A(j+1+k, j+1+2 n+1-k) \\
\leq & \frac{1}{2^{2 n+1}} \sum_{k=0}^{n} C(2 n, k) \frac{2 n-2 k+1}{2 n-k+1}(A(j+k, j+2 n+2-k) \\
& +A(j+k+1, j+2 n+1-k)) \\
= & \frac{1}{2^{2 n+1}}\left(A(j, j+2 n+2)+\sum_{k=1}^{n}\left(C(2 n, k) \frac{2 n-2 k+1}{2 n-k+1}\right.\right. \\
& \left.+C(2 n, k-1) \frac{2 n-2 k+3}{2 n-k+2}\right) A(j+k, j+2 n+2-k) \\
& \left.+C(2 n, n) \frac{1}{n+1} A(j+n+1, j+n+1)\right) \\
= & \frac{1}{2^{2 n+1}}\left(A(j, j+2 n+2)+\sum_{k=1}^{n} C(2 n+1, k) \frac{2 n-2 k+2}{2 n-k+2} A(j+k, j+2 n+2-k)\right) \\
= & \frac{1}{2^{2 n+1}} \sum_{k=0}^{n} C(2 n+1, k) \frac{2 n-2 k+2}{2 n-k+2} A(j+k, j+2 n+2-k) .
\end{aligned}
$$

Hence (iii) holds provided (ii) holds. We also have

$$
\begin{aligned}
& A(j+2 n+2, j+2 n+3) \\
&= A(j+1+2 n+1, j+1+2 n+2) \\
& \leq \frac{1}{2^{2 n+1}} \sum_{k=0}^{n} C(2 n+1, k) \frac{2 n-2 k+2}{2 n-k+2} A(j+1+k, j+1+2 n+2-k) \\
& \leq \frac{1}{2^{2 n+2}} \sum_{k=0}^{n} C(2 n+1, k) \frac{2 n-2 k+2}{2 n-k+2}(A(j+k, j+2 n+3-k) \\
&+A(j+k+1, j+2 n+2-k)) \\
&= \frac{1}{2^{2 n+2}}\left(A(j, j+2 n+3)+\sum_{k=1}^{n}\left(C(2 n+1, k) \frac{2 n-2 k+2}{2 n-k+2}\right.\right.
\end{aligned}
$$




$$
\begin{aligned}
& \left.+C(2 n+1, k-1) \frac{2 n-2 k+4}{2 n-k+3}\right) A(j+k, j+2 n+3-k) \\
& \left.+C(2 n+1, n) \frac{2}{n+2} A(j+n+1, j+n+2)\right) \\
& =\frac{1}{2^{2 n+2}}\left(A(j, j+2 n+3)+\sum_{k=1}^{n} C(2 n+2, k) \frac{2 n-2 k+3}{2 n-k+3} A(j+k, j+2 n+3-k)\right. \\
& \left.+C(2 n+2, n+1) \frac{1}{n+2} A(j+n+1, j+n+2)\right) \\
& =\frac{1}{2^{2 n+2}} \sum_{k=0}^{n+1} C(2 n+2, k) \frac{2 n-2 k+3}{2 n-k+3} A(j+k, j+2 n+3-k) .
\end{aligned}
$$

Thus (ii) holds when $n:=n+1$. By induction, we obtain (ii) and (iii). We put

$$
\alpha_{n}=\frac{1}{2^{2 n}} \sum_{k=0}^{n} C(2 n, k) \frac{2 n-2 k+1}{2 n-k+1}
$$

and

$$
\beta_{n}=\frac{1}{2^{2 n+1}} \sum_{k=0}^{n} C(2 n+1, k) \frac{2 n-2 k+2}{2 n-k+2} .
$$

From the above proof, we have $\beta_{n}=\alpha_{n}-2^{-2 n-1} C(2 n, n) /(n+1)$ and $\alpha_{n+1}=\beta_{n}$; and hence $\alpha_{n+1}=\alpha_{n}-2^{-2 n-1} C(2 n, n) /(n+1)$. We shall show by induction

$$
\alpha_{n}=2^{-2 n} C(2 n, n)
$$

for $n \in \mathbb{N} \cup\{0\}$. When $n=0$, (7) obviously holds. We assume (7) holds for some $n \in \mathbb{N} \cup\{0\}$. Then we have

$$
\begin{aligned}
\alpha_{n+1} & =\alpha_{n}-2^{-2 n-1} C(2 n, n) /(n+1) \\
& =2^{-2 n-2} C(2 n, n)(4-2 /(n+1)) \\
& =2^{-2 n-2} \frac{(2 n) !}{(n !)^{2}} \frac{4 n+2}{n+1}=2^{-2 n-2} \frac{(2 n) !}{(n !)^{2}} \frac{2 n+1}{n+1} \frac{2 n+2}{n+1} \\
& =2^{-2 n-2} C(2 n+2, n+1) .
\end{aligned}
$$

Thus (7) holds when $n:=n+1$. By induction, (7) holds for all $n$. We have

$$
\begin{aligned}
\lim _{n \rightarrow \infty} \alpha_{n} & =\lim _{n \rightarrow \infty} \frac{1}{2^{2 n}} \frac{(2 n) !}{(n !)^{2}} \\
& =\lim _{n \rightarrow \infty} \frac{1}{2^{2 n}} \frac{\frac{(2 n) !}{\sqrt{4 n}(2 n / e)^{2 n}} \sqrt{4 \pi n}(2 n / e)^{2 n}}{\left(\frac{n !}{\sqrt{2 \pi n}(n / e)^{n}}\right)^{2} 2 \pi n(n / e)^{2 n}} \\
& =\lim _{n \rightarrow \infty} \frac{\frac{(2 n) !}{\sqrt{2 \pi n}(2 n / e)^{2 n}}}{\left(\frac{n !}{\sqrt{2 \pi n}(n / e)^{n}}\right)^{2}} \frac{1}{\sqrt{\pi n}} \\
& =0 .
\end{aligned}
$$


We also have $\lim _{n} \beta_{n}=\lim _{n} \alpha_{n+1}=0$. By (i) and (ii), we obtain

$$
\begin{aligned}
& \lim _{n \rightarrow \infty} A(2 n, 2 n+1) \\
& \leq \lim _{n \rightarrow \infty} \frac{1}{2^{2 n}} \sum_{k=0}^{n} C(2 n, k) \frac{2 n-2 k+1}{2 n-k+1} A(k, 2 n+1-k) \\
& \leq \lim _{n \rightarrow \infty} \frac{1}{2^{2 n}} \sum_{k=0}^{n} C(2 n, k) \frac{2 n-2 k+1}{2 n-k+1} \\
& \quad=\lim _{n \rightarrow \infty} \alpha_{n}=0 .
\end{aligned}
$$

Similarly we can prove $\lim _{n} A(2 n+1,2 n+2)=0$. We have shown (iv).

A mapping $T$ on $C$ is said to be asymptotically regular at $x \in C[10]$ if

$$
\lim _{n \rightarrow \infty}\left\|T^{n+1} x-T^{n} x\right\|=0
$$

$T$ is said to be asymptotically regular on $C$ if $T$ is asymptotically regular at all $x \in C$.

Lemma 12 Let $T$ be a Chatterjea mapping on a subset $C$ of a Banach space E. Assume $\left\{T^{n} x\right\}$ is bounded for some $x \in C$. Then $T$ is asymptotically regular at $x$.

Proof Let $\eta$ satisfy (5). From the assumption, there exists a positive real number $M$ such that $M>\eta\left(2\left\|T^{n} x\right\|\right)$ for $n \in \mathbb{N} \cup\{0\}$. Define a function $A$ by

$$
A(m, n)=\frac{1}{M} \eta\left(\left\|T^{m} x-T^{n} x\right\|\right)
$$

for $m, n \in \mathbb{N} \cup\{0\}$ with $m \leq n$. Then all the assumption of Lemma 11 are satisfied. So we obtain

$$
\lim _{n \rightarrow \infty} \eta\left(\left\|T^{n} x-T^{n+1} x\right\|\right)=M \lim _{n \rightarrow \infty} A(n, n+1)=0
$$

Therefore $T$ is asymptotically regular at $x$.

Proposition 13 Let $T$ be a Chatterjea mapping on a subset $C$ of a Banach space E. Assume $\left\{T^{n} u\right\}$ is bounded for some $u \in C$. Then the following hold:

(i) $\left\{T^{n} x\right\}$ is bounded for all $x \in C$.

(ii) $T$ is asymptotically regular on $C$.

Proof Let $\eta$ satisfy (5). Define a continuous function $f$ from $C$ into $[0, \infty)$ by

$$
f(x)=\limsup _{n \rightarrow \infty} \eta\left(\left\|T^{n} u-x\right\|\right)
$$

for all $x \in C$. Then $f$ is well defined from the assumption. We have

$$
\begin{aligned}
2 f(T x) & =\limsup _{n \rightarrow \infty} 2 \eta\left(\left\|T^{n} u-T x\right\|\right) \\
& \leq \limsup _{n \rightarrow \infty}\left(\eta\left(\left\|T^{n} u-x\right\|\right)+\eta\left(\left\|T^{n-1} u-T x\right\|\right)\right)
\end{aligned}
$$




$$
\begin{aligned}
& \leq \limsup _{n \rightarrow \infty} \eta\left(\left\|T^{n} u-x\right\|\right)+\limsup _{n \rightarrow \infty} \eta\left(\left\|T^{n-1} u-T x\right\|\right) \\
& =f(T x)+f(x),
\end{aligned}
$$

which implies $f(T x) \leq f(x)$. Thus, $f$ is nonincreasing with respect to $T$. Hence $f\left(T^{n} x\right) \leq$ $f(x)$ for $n \in \mathbb{N}$. This implies that $\left\{T^{n} x\right\}$ is bounded. We have shown (i). By Lemma 12, we obtain (ii).

\section{Convergence theorems}

In this section, we prove convergence theorems under the assumption that the domain $C$ has the Opial property.

Proposition 14 Let $T$ be a Chatterjea mapping on a subset $C$ of a Banach space E. Assume $C$ has the Opial property. If $\left\{x_{n}\right\}$ converges weakly to $z \in C$ and $\lim _{n}\left\|T x_{n}-x_{n}\right\|=0$, then $T z=z$. That is, $I-T$ is demiclosed at zero.

Proof We note that $\left\{x_{n}\right\}$ is bounded. Since

$$
\left\|T x_{n}-y\right\|-\left\|T x_{n}-x_{n}\right\| \leq\left\|x_{n}-y\right\| \leq\left\|T x_{n}-x_{n}\right\|+\left\|T x_{n}-y\right\|,
$$

we have

$$
\limsup _{n \rightarrow \infty}\left\|T x_{n}-y\right\|=\limsup _{n \rightarrow \infty}\left\|x_{n}-y\right\|
$$

for all $y \in C$. Using this, we have

$$
\begin{aligned}
2 \eta\left(\limsup _{n \rightarrow \infty}\left\|x_{n}-T z\right\|\right) & =2 \eta\left(\limsup _{n \rightarrow \infty}\left\|T x_{n}-T z\right\|\right) \\
& =\limsup _{n \rightarrow \infty} \eta\left(\left\|T x_{n}-T z\right\|\right) \\
& \leq \limsup _{n \rightarrow \infty}\left(\eta\left(\left\|T x_{n}-z\right\|\right)+\eta\left(\left\|x_{n}-T z\right\|\right)\right) \\
& \leq \limsup _{n \rightarrow \infty} \eta\left(\left\|T x_{n}-z\right\|\right)+\limsup _{n \rightarrow \infty} \eta\left(\left\|x_{n}-T z\right\|\right) \\
& =\eta\left(\limsup _{n \rightarrow \infty}\left\|T x_{n}-z\right\|\right)+\eta\left(\limsup _{n \rightarrow \infty}\left\|x_{n}-T z\right\|\right) \\
& =\eta\left(\limsup _{n \rightarrow \infty}\left\|x_{n}-z\right\|\right)+\eta\left(\limsup _{n \rightarrow \infty}\left\|x_{n}-T z\right\|\right)
\end{aligned}
$$

and hence

$$
\eta\left(\limsup _{n \rightarrow \infty}\left\|x_{n}-T z\right\|\right) \leq \eta\left(\limsup _{n \rightarrow \infty}\left\|x_{n}-z\right\|\right)
$$

Since $C$ has the Opial property, we obtain $T z=z$.

Remark A function $y \mapsto \lim \sup _{n} \eta\left(\left\|x_{n}-y\right\|\right)$ from $C$ into $[0, \infty)$ is also nonincreasing with respect to $T$. 
Theorem 15 Let $T$ be a Chatterjea mapping on a subset $C$ of a Banach space E. Assume $\left\{T^{n} u\right\}$ is bounded for some $u \in C$; and $C$ is boundedly weakly compact and has the Opial property. Then $\left\{T^{n} x\right\}$ converges weakly to a fixed point of $T$ for all $x \in C$.

Remark We do not need the convexity of $C$.

Proof Fix $x \in C$. By Proposition 13, $\left\{T^{n} x\right\}$ is bounded and $\lim _{n}\left\|T^{n} x-T \circ T^{n} x\right\|=0$. From the assumption, there exist a subsequence $\left\{T^{n_{j}} x\right\}$ of $\left\{T^{n} x\right\}$ and $z \in C$ such that $\left\{T^{n_{j}} x\right\}$ converges weakly to $z$. By Proposition $14, z$ is a fixed point of $T$. By Proposition 8, we note that $\left\{\left\|T^{n} x-z\right\|\right\}$ is a nonincreasing sequence. Arguing by contradiction, assume that $\left\{T^{n} x\right\}$ does not converge to $z$. Then there exist a subsequence $\left\{T^{n_{k}} x\right\}$ of $\left\{T^{n} x\right\}$ and $w \in C$ such that $\left\{T^{n_{k}} x\right\}$ converges weakly to $w$ and $z \neq w$. We note $T w=w$. From the Opial property,

$$
\begin{aligned}
\lim _{n \rightarrow \infty}\left\|T^{n} x-z\right\| & =\lim _{j \rightarrow \infty}\left\|T^{n_{j}} x-z\right\|<\lim _{j \rightarrow \infty}\left\|T^{n_{j}} x-w\right\|=\lim _{n \rightarrow \infty}\left\|T^{n} x-w\right\| \\
& =\lim _{k \rightarrow \infty}\left\|T^{n_{k}} x-w\right\|<\lim _{k \rightarrow \infty}\left\|T^{n_{k}} x-z\right\|=\lim _{n \rightarrow \infty}\left\|T^{n} x-z\right\| .
\end{aligned}
$$

This is a contradiction. Therefore $\left\{T^{n} x\right\}$ converges weakly to $z$.

As direct consequences of Theorem 15, we obtain the following.

Corollary 16 Let $T$ be a Chatterjea mapping on a weakly compact subset $C$ of a Banach space E. Assume $C$ has the Opial property. Then $\left\{T^{n} x\right\}$ converges weakly to a fixed point of $T$ for all $x \in C$.

Corollary 17 Let $T$ be a Chatterjea mapping on a compact subset $C$ of a Banach space $E$. Then $\left\{T^{n} x\right\}$ converges strongly to a fixed point of $T$ for all $x \in C$.

\section{Existence theorems}

In this section, we prove the existence of fixed points of Chatterjea mappings. By Lemma 9 and Theorem 15, we obtain the following.

Theorem 18 Let $T$ be a Chatterjea mapping on a subset $C$ of a Banach space E. Assume $C$ is boundedly weakly compact and has the Opial property. Then the following are equivalent:

(i) $\left\{T^{n} u\right\}$ is bounded for some $u \in C$.

(ii) $T$ has a fixed point.

As direct consequences of Theorem 18, we obtain the following.

Corollary 19 Let $T$ be a Chatterjea mapping on a subset $C$ of a Banach space E. Assume that either of the following holds:

- $C$ is compact;

- $C$ is weakly compact and has the Opial property.

Then $T$ has a fixed point.

Remark It is obvious that Corollary 19 also can be proved by Corollaries 16 and 17. 
Corollary 20 Let $T$ be a nonspreading mapping on a weakly closed subset $C$ of a Hilbert space $E$. Then the following are equivalent:

(i) $\left\{T^{n} u\right\}$ is bounded for some $u \in C$.

(ii) $T$ has a fixed point.

Remark Corollary 20 is a generalization of Corollary 4.4 in [1] because we do not assume the convexity of $C$.

We shall prove fixed point theorems in UCED Banach spaces.

Lemma 21 Let $C$ be a boundedly weakly compact and convex subset of a Banach space $E$. Let $T$ be a mapping on a subset $C$. Assume that there exists a lower semicontinuous, strictly quasiconvex function $f$ from $C$ into $\mathbb{R}$ such that $f$ is nonincreasing with respect to $T$ and $f$ satisfies (4). Then $T$ has a fixed point.

Proof Since $f$ is quasiconvex, we note that $f$ is lower semicontinuous in the weak topology. By Lemma 2, there exists $z \in C$ such that $f(z)=\inf f(C)$. Since $f$ is nonincreasing with respect to $T, f(T z) \leq f(z)$. Thus $f(T z)=f(z)$. If $T z \neq z$, then since $f$ is strictly quasiconvex, we have

$$
f(z) \leq f((z+T z) / 2)<\max \{f(z), f(T z)\}=f(z) .
$$

This is a contradiction. Hence $T z=z$.

Lemma 22 Let $C$ be a boundedly weakly compact and convex subset of a Banach space $E$. Let $T_{0}, T_{1}, T_{2}, \ldots, T_{\ell}$ be commuting mappings on $C$. Assume that for every $j=0,1,2, \ldots, \ell$, there exists a lower semicontinuous, strictly quasiconvex function $f_{j}$ from $C$ into $\mathbb{R}$ such that $f_{j}$ is nonincreasing with respect to $T_{j}$ and $f_{j}$ satisfies (4). Assume also that $F\left(T_{j}\right)$ is closed and convex for $j=1,2, \ldots, \ell$. Then $\bigcap_{j=0}^{\ell} F\left(T_{j}\right)$ is nonempty.

Proof By Lemma 21, $F\left(T_{1}\right)$ is nonempty. Since $F\left(T_{1}\right)$ is closed and convex, $F\left(T_{1}\right)$ is weakly closed. Thus $F\left(T_{1}\right)$ is boundedly weakly compact. We assume that $A_{k-1}:=\bigcap_{j=1}^{k-1} F\left(T_{j}\right)$ is nonempty, boundedly weakly compact and convex for some $k \in \mathbb{N}$ with $1<k \leq \ell$. For $x \in$ $A_{k-1}$ and $j \in \mathbb{N}$ with $1 \leq j<k$, since $T_{k} \circ T_{j}=T_{j} \circ T_{k}$, we have $T_{k} x=T_{k} \circ T_{j} x=T_{j} \circ T_{k} x$, thus $T_{k} x$ is a fixed point of $T_{j}$. Therefore $T_{k}\left(A_{k-1}\right) \subset A_{k-1}$. By Lemma 21 again, $T_{k}$ has a fixed point in $A_{k-1}$, thus, $A_{k}:=\bigcap_{j=1}^{k} F\left(T_{j}\right) \neq \varnothing$. Since $A_{k}$ is closed and convex, $A_{k}$ is nonempty, boundedly weakly compact and convex. By induction, $A_{\ell}$ is nonempty, boundedly weakly compact and convex. By Lemma 21, $T_{0}$ has a fixed point in $A_{\ell}$. This completes the proof.

Lemma 23 Let $C$ be a weakly compact and convex subset of a Banach space E. Let $S=$ $\left\{T_{0}\right\} \cup S^{\prime}$ be a family of commuting mappings on $C$. Assume that for every $T \in S$, there exists a lower semicontinuous, strictly quasiconvex function $f_{T}$ from $C$ into $\mathbb{R}$ such that $f_{T}$ is nonincreasing with respect to $T$. Assume also that $F(T)$ is closed and convex for $T \in S^{\prime}$. Then $S$ has a common fixed point.

Proof By Lemma 22, $\left\{F(T): T \in S^{\prime}\right\}$ has the finite intersection property. Since $C$ is weakly compact and $F(T)$ is weakly closed for every $T \in S^{\prime}$, we have $A:=\bigcap_{T \in S^{\prime}} F(T) \neq \varnothing$. Since $A$ 
is weakly compact and $T_{0}(A) \subset A, T_{0}$ has a fixed point in $A$. Thus $S$ has a common fixed point.

Lemma 24 Let $C$ be a convex subset of a UCED Banach space E. Let $T$ be a Chatterjea mapping on $C$. Assume that $\left\{T^{n} u\right\}$ is bounded for some $u \in C$. Define a function $f$ from $C$ into $[0, \infty)$ by (9). Then $f$ is a continuous, strictly quasiconvex function such that $f$ is nonincreasing with respect to $T$ and $f$ satisfies (4).

Proof We note that a function $g$ defined by (3) is continuous and strictly quasiconvex; and $g$ satisfies (4). So $f$ is also continuous and strictly quasiconvex; and $f$ satisfies (4). We have shown that $f$ is nonincreasing with respect to $T$ in the proof of Proposition 13.

Using Lemmas 21-24, we obtain the following.

Theorem 25 Let $C$ be a boundedly weakly compact and convex subset of a UCED Banach space E. Let $T$ be a Chatterjea mapping on $C$. Then the following are equivalent:

(i) $\left\{T^{n} u\right\}$ is bounded for some $u \in C$.

(ii) $T$ has a fixed point.

Theorem 26 Let $C$ be a boundedly weakly compact and convex subset of a UCED Banach space E. Let $T_{1}, T_{2}, \ldots, T_{\ell}$ be commuting Chatterjea mappings on C. Assume that $\left\{T_{j}^{n} u\right\}$ is bounded for all $u \in C$ and $j$. Then $\bigcap_{j=1}^{\ell} F\left(T_{j}\right)$ is nonempty.

Theorem 27 Let $C$ be a weakly compact and convex subset of a UCED Banach space E. Let $S$ be a family of commuting Chatterjea mappings on $C$. Then $S$ has a common fixed point.

Competing interests

The author did not provide this information.

\section{Acknowledgements}

The author is grateful to the referees for their careful reading. The author is supported in part by a Grant-in-Aid for Scientific Research from the Japan Society for the Promotion of Science.

Received: 31 October 2013 Accepted: 24 January 2014 Published: 21 Feb 2014

\section{References}

1. Kohsaka, F, Takahashi, W: Fixed point theorems for a class of nonlinear mappings related to maximal monotone operators in Banach spaces. Arch. Math. 91, 166-177 (2008). MR2430800

2. Chatterjea, SK: Fixed-point theorems. C. R. Acad. Bulgare Sci. 25, 727-730 (1972). MR0324493

3. Goebel, K, Kirk, WA: Topics in Metric Fixed Point Theory. Cambridge Studies in Advanced Mathematics, vol. 28. Cambridge University Press, Cambridge (1990). MR1074005

4. Prus, S: Geometrical background of metric fixed point theory. In: Kirk, WA, Sims, B (eds.) Handbook of Metric Fixed Point Theory, pp. 93-132. Kluwer Academic, Dordrecht (2001). MR1904275

5. Suzuki, T: Fixed point theorems and convergence theorems for some generalized nonexpansive mappings. J. Math. Anal. Appl. 340, 1088-1095 (2008). MR2390912

6. Opial, Z: Weak convergence of the sequence of successive approximations for nonexpansive mappings. Bull. Am. Math. Soc. 73, 591-597 (1967). MR0211301

7. Gossez, J-P, Lami Dozo, E: Some geometric properties related to the fixed point theory for nonexpansive mappings. Pac. J. Math. 40, 565-573 (1972). MR0310717

8. van Dulst, D: Equivalent norms and the fixed point property for nonexpansive mappings. J. Lond. Math. Soc. 25 139-144 (1982). MR0645871

9. Diaz, JB, Metcalf, FT: On the structure of the set of subsequential limit points of successive approximations. Bull. Am. Math. Soc. 73, 516-519 (1967). MR0211387

10. Browder, FE, Petryshyn, WV: The solution by iteration of nonlinear functional equations in Banach spaces. Bull. Am. Math. Soc. 72, 571-575 (1966). MR0190745 
10.1186/1687-1812-2014-47

Cite this article as: Suzuki: Fixed point theorems for a new nonlinear mapping similar to a nonspreading mapping Fixed Point Theory and Applications 2014, 2014:47

Submit your manuscript to a SpringerOpen ${ }^{\circ}$ journal and benefit from:

- Convenient online submission

- Rigorous peer review

- Immediate publication on acceptance

- Open access: articles freely available online

- High visibility within the field

- Retaining the copyright to your article

Submit your next manuscript at $\gg$ springeropen.com 